\title{
RATIONAL CURVES AND POINTS ON K3 SURFACES
}

\author{
By Fedor Bogomolov and Yuri Tschinkel
}

Abstract. We study the distribution of algebraic points on K3 surfaces.

1. Introduction. Let $k$ be a field and $\bar{k}$ a fixed algebraic closure of $k$. We are interested in connections between geometric properties of algebraic varieties and their arithmetic properties over $k$, over its finite extensions $k^{\prime} / k$ or over $\bar{k}$. Here we study certain varieties of intermediate type, namely $\mathrm{K} 3$ surfaces and their higher dimensional generalizations, Calabi-Yau varieties.

To motivate the following discussion, let $S$ be a K3 surface over $k$. In positive characteristic, $S$ may be unirational and covered by rational curves. Examples are supersingular K3 surfaces over fields of characteristic two or the surface

$$
x^{4}+y^{4}+z^{4}+t^{4}=0
$$

over fields of characteristic three. If $k$ has characteristic zero, then $S$ contains at most finitely many rational curves in each homology class of $S$ (the counting of which is an interesting problem in enumerative geometry, see [4], [6], [7], [26]). Over uncountable fields, there may, of course, exist $k$-rational points on $S$ not contained in any rational curve defined over $\bar{k}$. The following extremal statement, proposed by the first author in 1981, is however still a logical possibility:

Let $k$ be either a finite field or a number field. Let $S$ be a $K 3$ surface defined over $k$. Then every $\bar{k}$-rational point on $S$ lies on some rational curve $C \subset S$, defined over $\bar{k}$.

In this note we collect several representative examples illustrating this statement. One of our results is:

THEOREM 1.1. Let $S$ be a Kummer surface over a finite field $k$. Then every $s \in S(\bar{k})$ lies on a rational curve $C \subset S$ defined over $\bar{k}$.

Manuscript received October 6, 2003; revised November 10, 2004.

American Journal of Mathematics 127 (2005), 825-835. 
Actually, such surfaces $S$ are rationally connected in a very strong sense: there is a Zariski open subset $S^{0} \subset S$ such that for every finite set of points $\left\{s_{1}, \ldots, s_{n}\right\} \subset S^{0}(\bar{k})$ there is a (singular) irreducible rational curve $C \subset S$ defined over $\bar{k}$ which contains $s_{j}$, for all $j$. If $S$ is not supersingular, then $S$ is not uniruled. This resolves a problem raised by Katsura in [9], Question 12, and a question of Kollár in [2], Remark 12.

Using this theorem we produce examples of nonuniruled surfaces of general type (with nontrivial unramified Brauer groups) over finite fields which are "rationally chain connected" (any two algebraic points can be joined by a chain of rational curves).

Acknowledgments. We are grateful to Brendan Hassett, Ching-Li Chai, Nick Katz, Barry Mazur and Bjorn Poonen for their interest and comments. We are very indebted to the referee for many comments which helped to improve the exposition.

2. Preliminaries: abelian varieties. In this section we collect some facts concerning abelian varieties. Our basic reference is [14].

Let $A$ be an abelian variety over $\bar{k}$. Let $A[n] \subset A(\bar{k})$ be the set of the $n$-torsion points of $A$. If $k$ is finite, then every point in $A(\bar{k})$ is a torsion point. For every torsion point $x \in A(\bar{k})$ let

$$
\operatorname{ord}(x):=\min \left\{n \in \mathbb{Z}_{>0} \mid n x=0\right\}
$$

be the order of $x$. Let $\operatorname{End}_{\bar{k}}(A)$ be the ring of $\bar{k}$-endomorphisms of $A$. Every abelian variety $A$ defined over $\bar{k}$ is isogenous to a product of simple abelian varieties (over $\bar{k}$ ).

An elliptic curve over a field $k$ of characteristic $p>0$ is called supersingular if its $p$-rank is zero, and an abelian variety over $k$ is called supersingular if it is $\bar{k}$-isogenous to a product of supersingular elliptic curves.

Remark 2.1. In our applications, we will use hyperelliptic curves contained in abelian varieties. Over an algebraically closed field, every (principally polarized) abelian surface is the Jacobian of a (possibly reducible) hyperelliptic curve (see [25]). This fails in higher dimensions: a generic principally polarized abelian variety of dimension $\geq 3$ over $\mathbb{C}$ does not contain hyperelliptic curves [17]. A similar result holds over large fields of positive characteristic, such as an algebraic closure of $\overline{\mathbb{F}}_{q}(t)$ [16]. It could still be that over an algebraic closure of a finite field, every abelian variety of dimension $\geq 2$ contains a hyperelliptic curve.

Let $C$ be a smooth projective geometrically connected curve of genus $\mathrm{g}=$ $g(C) \geq 2$ over a field $k$. Let $J=J_{C}$ be the Jacobian of $C$. Throughout, we assume that $C(k) \neq \emptyset$ and choose a point $c_{0} \in C(k)$ which we use to identify the degree 
$n$ Jacobian $J^{(n)}$ with $J$ and to embed $C$ in $J$. Consider the maps

$$
\begin{gathered}
C^{n} \longrightarrow \phi_{n} \operatorname{Sym}^{(n)}(C) \stackrel{\varphi_{n}}{\longrightarrow} J^{(n)}(C), \\
c=\left(c_{1}, \ldots, c_{n}\right) \longrightarrow\left(c_{1}+\cdots+c_{n}\right) \longrightarrow[c],
\end{gathered}
$$

Here $\left(c_{1}+\cdots+c_{n}\right)$ denotes the zero-cycle. The map $\phi_{n}$ is a finite cover of degree $n$ !. For all $n \geq 2 g+1$, the map $\varphi_{n}$ is a $\mathbb{P}^{n-9}$-bundle and the map $C^{n} \rightarrow J^{(n)}(C)$ is surjective with geometrically irreducible fibers (see [13], Corollary 9.1.4, for example). For $x \in J(k)=J^{(n)}(k)$ put $\mathbb{P}_{x}:=\varphi_{n}^{-1}(x) \subset \operatorname{Sym}^{(n)}(C)$.

LEMMA 2.2. Let $C$ be a smooth projective geometrically connected curve over $\mathbb{F}_{q}$ of genus $g=g(C) \geq 2$, with Jacobian J. For every point $x \in J\left(\mathbb{F}_{q}\right)$ and every $n \geq 2 g+1$ there exist a finite extension $k / \mathbb{F}_{q}$ and a point $y \in \mathbb{P}_{x}(k)$ such that the degree $n$ zero-cycle $c_{1}+\cdots+c_{n}$ on $C$ corresponding to $y$ is $k$-irreducible.

Proof. Let $x \in J\left(\mathbb{F}_{q}\right)$ be a point and $\mathbb{P}_{x}=\varphi_{n}^{-1}(x)$ the fiber over $x$. The restriction $\phi_{n, x}$ of $\phi_{n}$ to $\mathbb{P}_{x}$ is a cover of degree $n !$.

We apply an equidistribution theorem of Deligne as in [13]. Let $k / \mathbb{F}_{q}$ be a finite extension. In the terminology of [13], Theorem 9.4.4, let $T=\operatorname{Spec}\left(\mathbb{F}_{q}\right)$ and put $t=\operatorname{Spec}(k),(t$ is a $k$-valued point of $T)$. Let $E / k$ be the (unique) degree $n$ extension and $X_{t, \text { prime }}(E)$ the subset of $E$-valued points of a natural $\mathbb{G}_{m}$-bundle $X_{t}$ over a Zariski open subvariety of the fiber $\left(\varphi_{n} \circ \phi_{n}\right)^{-1}(x) \subset C^{n}$, defined in [13], p. 189. The image of $X_{t, \text { prime }}(E)$ in $C^{n}(E)$ consists of $n$-tuples of distinct points $\left(c_{1}, \ldots, c_{n}\right)$ such that the Galois group $\operatorname{Gal}(E / k)$ acts transitively on the set $\left\{c_{1}, \ldots, c_{n}\right\}$. By Theorem 9.4.4 in [13], there exist constants $a(x)=a(x, T)$ and $c(x)=c(x, T)$ such that for any $k$ with $\operatorname{Card}(k) \geq a(x)$ one has

$$
\left|\# X_{t, \text { prime }}(E) / \# X_{t}(E)-1 / n\right| \leq c(x) n ! /(\# E)^{1 / 2} .
$$

Note that $\# E=\operatorname{Card}(k)^{n}$. By effective Weil estimates as in Theorem 9.1.2 of [13], $X_{t}(E) \neq \emptyset$; combining this with the inequality (2.1) we find that for $k$ sufficiently large $\# X_{t, \text { prime }}(E) \neq \emptyset$, as claimed.

Remark 2.3. A similar result has been used in [18], Lemma 5.

COROLlaRY 2.4. Let $C$ be a curve of genus $g(C) \geq 2$ over a (sufficiently large) finite field $k$, let $J$ be its Jacobian and $x \in J(k)$ a point. Choose a point $c_{0}$ on $C(k)$ and use it to identify $J$ with $J^{(n)}$, for all $n$, and to embed $C$ in $J$. For every $n \geq 2 g+1$ there exist a point $c \in C(E)$, where $E / k$ is the (unique) extension of $k$ of degree $n$, and an endomorphism $\Phi=\Phi_{n} \in \operatorname{End}_{k}(J)$ such that $\Phi(c)=x$.

Proof. For any $n \geq 2 g(C)+1$ consider the surjective map $\varphi_{n}$. Let $x \in J^{(n)}(k)$ be a point and let $\mathbb{P}_{x}$ be the projective space over $x$. Extending $k$, if necessary, 
we find a $y \in \mathbb{P}_{x}(k)$ such that the zero-cycle $\left(c_{1}+\cdots+c_{n}\right)$ corresponding to $y$ is irreducible over $k$, by Lemma 2.2.

We have $y=\sum_{g \in G} c^{g}$, with $c:=c_{1} \in C(E)$, where $E / k$ is the unique extension of $k$ of degree $n$ and $G:=\operatorname{Gal}(E / k)$. The group $G$ is cyclic, generated by the Frobenius automorphism, which we denote by Fr. Thus

$$
y=\sum_{j=0}^{n-1} \operatorname{Fr}^{j}(c) .
$$

The Frobenius morphism "lifts" to an endomorphism of $J$, that is, there exists an endomorphism $\tilde{\mathrm{Fr}} \in \operatorname{End}_{k}(J)$ which acts on $J(E)$ in the same way as the Galois automorphism Fr $\in \operatorname{Gal}(E / k)$. Put

$$
\Phi:=\sum_{j=0}^{n-1} \tilde{\mathrm{Fr}}^{j}
$$

as an element of $\operatorname{End}_{k}(J)$.

Remark 2.5. In particular, Corollary 2.4 implies that if $\operatorname{ord}(x)=m$ then there exist infinitely many points in $C(\bar{k}) \subset J(\bar{k})$ whose order is divisible by $m$. Indeed, notice that $\operatorname{ord}(c)=\operatorname{ord}\left(c^{g}\right)$, for all $g \in \operatorname{Gal}(E / k)$. Since the order of $x$ is $m$ the order $\operatorname{ord}(c)$ is divisible by $m$.

A related result has been proved in [1]: Let $\ell$ be a prime, $C$ a curve (defined over a finite field $k$ ), $J$ its Jacobian, $C \subset J$ an Albanese embedding and $\lambda: J(\bar{k}) \rightarrow$ $J(\bar{k})_{\ell}$ the projection onto the $\ell$-primary part. Then the map $\lambda: C(\bar{k}) \rightarrow J(\bar{k})_{\ell}$ is surjective. It was noticed in [19], p. 112, that the method of [1] can be used to prove that any positive-dimensional subvariety of a geometrically simple abelian variety (over a finite field) contains infinitely many points of pairwise prime orders.

The argument in the proof of Corollary 2.4 gives a statement very much in the spirit of [12]:

COROLLARY 2.6. Let $C$ be a curve of genus g over a sufficiently large finite field $k$ and $J$ its Jacobian. Then there exist a morphism $\lambda: C \rightarrow J$ (depending on $k$ ) and a field $E / k$ such that $J(k) \subset \lambda(C(E))$.

LEMMA 2.7. Let $K$ be a number field (or any field where Hilbert's irreducibility holds). Let $C$ be a curve of genus $g=g(C) \geq 2$ over $K$ and $J$ its Jacobian. Assume that $C$ has a point $c_{0} \in C(K)$ and use this point to identify $J^{(n)}=J$ and the embedding $C \rightarrow J$. For any point $x \in J(K)$ and any $n \geq 2 g+1$ there exist an extension $K^{\prime} / K$ of degree $n$ and a point $c \in C\left(K^{\prime}\right)$ such that the cycle $\operatorname{Tr}_{K^{\prime} / K}(c)$ equals $x \in J^{(n)}(K)=J(K)$. 
Proof. The inverse image of $\mathbb{P}_{x}$ under $C^{n} \rightarrow \operatorname{Sym}^{(n)}(C)$ is a geometrically irreducible, generically Galois cover of $\mathbb{P}_{x}$ (see Corollary 9.1.4 in [13], for example). Hilbert's irreducibility theorem (as in [22], Proposition 2 in Section 9.2 and "Hilbert's theorem" in Section 9.6) implies the claim.

3. Preliminaries: K3 surfaces. In this section we assume that the ground field $k$ is algebraically closed. A good general reference for the following material is [21] and [8].

Definition 3.1. A smooth connected simply-connected projective algebraic surface with trivial canonical class is called a K3 surface. A K3 surface $S$ with $\operatorname{rk} \operatorname{Pic}(S)=22$ is called supersingular.

Example 3.2. Examples of $\mathrm{K} 3$ surfaces are double covers of $\mathbb{P}^{2}$ ramified in a smooth curve of degree 6 , smooth quartic hypersurfaces in $\mathbb{P}^{3}$ or smooth intersections of 3 quadrics in $\mathbb{P}^{5}$.

Another interesting series of examples is given by (generalized) Kummer surfaces: desingularizations of quotients of abelian surfaces by certain finite group actions (see Proposition 4.4).

Remark 3.3. If $S$ is a $\mathrm{K} 3$ surface over a field of characteristic zero, then rk $\operatorname{Pic}(S) \leq 20$. An example of a supersingular $S$ over a field of positive characteristic is given by a desingularization of $A / \sigma$, where $A$ is a supersingular abelian surface and $\sigma$ the standard involution (multiplication by -1 map).

Remark 3.4. If $S$ is uniruled then the Brauer group of $S$ has trivial transcendental part, and all cycles are algebraic. This implies that $\operatorname{rk} \operatorname{Pic}(S)=22$ (i.e., $S$ is supersingular). In particular, this is possible only in positive characteristic [21], [3].

In characteristic 2, every supersingular K3 surface is unirational [20]. It is conjectured that all supersingular K3 surfaces are unirational (see [21], Section 5, or [9], Problem 12). A generalized Kummer surface $S \sim A / G$ is uniruled iff it is unirational iff the corresponding abelian surface $A$ is supersingular [23], [11].

4. Construction. Unless stated otherwise, the ground field $k$ is algebraically closed of characteristic $\neq 2$. We recall the classical construction of special K3 surfaces, called Kummer surfaces. Let $A$ be an abelian surface,

$$
\begin{aligned}
\sigma: A & \rightarrow A \\
a & \mapsto-a
\end{aligned}
$$

the standard involution. The set of fixed points of $\sigma$ is exactly $A[2]$. The blowup $S:=\widehat{A / \sigma}$ of the image of $A[2]$ in the quotient $A / \sigma$ is a smooth K3 surface $S$, called a Kummer surface:

$$
A / \sigma \rightarrow S, \quad \widehat{A / \sigma} \rightarrow S .
$$


LEMMA 4.1. Rational curves $C$ in $A / \sigma$ correspond to hyperelliptic curves $\tilde{C} \subset$ A containing a point $P \in A[2]$ and such that the hyperelliptic involution on $\tilde{C}$ coincides with $\sigma$.

Proof. The hyperelliptic involution on $\tilde{C}$ acts as an involution $\sigma: x \rightarrow-x$ on the Jacobian $J=J_{\tilde{C}}$ and hence also on the abelian subvariety which is the image of $J$ in $A$. In particular, the involution $\sigma$ on $A$ induces the standard hyperelliptic involution on $C$. Hence $C / \sigma$ is rational and defines a rational curve in $A / \sigma$. Conversely, if $C \in A / \sigma$ is rational then the preimage of $C$ in $A$ is irreducible (since $A$ doesn't contain rational curves). Thus $C=\tilde{C} / \sigma$ and $\tilde{C}$ is hyperelliptic and all ramification points of the map $\tilde{C} \rightarrow C$ are contained among the two-torsion points $A[2] \cap \tilde{C}$.

THEOREM 4.2. Let $S$ be a Kummer surface over a finite field $k, C$ a curve of genus 2 defined over $k, J$ its Jacobian and $S \sim J / \sigma$ the associated Kummer surface. Then every algebraic point $s \in S(\bar{k})$ lies on some rational curve, defined over $\bar{k}$.

Proof. Let $s \in S(\bar{k})$ be an algebraic point (on the complement to the 16 exceptional curves) and $x \in J(\bar{k})$ one of its preimages. We have proved in Corollary 2.4 that for every $x \in J(\bar{k})$ (and any Albanese embedding $C \rightarrow J$ ) there is an endomorphism $\Phi \in \operatorname{End}_{\bar{k}}(J)$ such that $\Phi \cdot C(\bar{k})$ contains $x$ (note that $\Phi$ commutes with the involution $\sigma$ ). The image of the curve $\Phi \cdot C$ in $S$ contains $s$.

Combining Theorem 4.2 with Corollary 2.4 we obtain:

COROLLARY 4.3. Let $S$ be a Kummer surface over a finite field $k$. There are infinitely many rational curves (defined over $\bar{k}$ ) through every point in the complement of the 16 exceptional curves in $S(\bar{k})$. If $S$ is nonuniruled, these curves do not form an algebraic family.

In addition to quotients $A / \sigma$, there exist generalized Kummer $\mathrm{K} 3$ surfaces obtained as desingularizations of abelian surfaces under actions of other finite groups. Such actions have been classified:

Proposition 4.4. (see [11]) Let $A$ be an abelian surface over a field $k$ and $G$ a finite group acting on $A$ such that the quotient $A / G$ is birational to a $K 3$ surface. If $\operatorname{char}(k)>0$ then $G$ is one of the following:

- a cyclic group of order 2,3,4,5,6,8,10,12;

- a binary dihedral group $(2,2, n)$ with $n=2,3,4,5,6$;

- a binary tetrahedral group $(2,3,3)$;

- a binary octahedral group $(2,3,4)$;

- a binary icosahedral group $(2,3,5)$.

If $\operatorname{char}(k)=0$ then $G$ is one of the following:

- a cyclic group of order 2,3,4,6;

- a binary dihedral group $(2,2, n)$ with $n=2,3$;

- a binary tetrahedral group $(2,3,3)$. 
The groups listed above do indeed occur.

COROLLARY 4.5. If $S \sim A / G$ is a generalized Kummer K3 surface over a finite field $k$ (of characteristic $\geq 7$ ) then every algebraic point on $S$ lies on infinitely many rational curves, defined over $\bar{k}$.

Proof. By Remark 3.4, a supersingular generalized Kummer K3 surface is uniruled and the claim follows. By Lemma 6.2 in [11], if $S$ is not supersingular and $G$ is divisible by two then $G$ has a unique element of order two, acting as the standard involution. An argument as in the proof of Theorem 4.2 applies to show that every algebraic point lies on a rational curve. The generalized Kummer K3 surfaces with $G=\mathbb{Z} / 5$ are supersingular [11].

It remains to consider $G=\mathbb{Z} / 3$. In this case, the abelian variety $A$ is isogenous to $E \times E$ with an action of $\mathbb{Z} / 3$ which is obtained from the cyclic permutation action on $E^{3}$ divided by the diagonal. The quotient surface $A / \mathbb{Z} / 3$ is birationally equivalent to a $K 3$-surface (it is simply-connected, has a nontrivial holomorphic $(2,0)$-form and Kodaira dimension 0$)$. In order to apply our general argument we need to find a generating curve $C \in A$ with a rational quotient $C / \mathbb{Z} / 3$. Consider the action of $\mathfrak{S}_{3}$ on $\mathbb{P}^{1}$ with $\mathbb{Z} / 3$-invariant points $0, \infty$. Let $S$ be an $\mathfrak{S}_{3}$-orbit in $\mathbb{P}^{1}$ and $C_{S}$ the double cover of $\mathbb{P}^{1}$ ramified in $S$. Then $g\left(C_{S}\right)=2$ and $\mathfrak{S}_{3}$ acts on the hyperelliptic curve $C_{S}$. The automorphism group of $C_{S}$, for a generic orbit $S$, is equal to $\mathfrak{S}_{3} \times \mathbb{Z} / 2$. There is an action of $\mathfrak{S}_{3}$ on $J=J_{C_{S}}$, note that $J$ is isogenous to $E \times E$. For any subgroup $\mathbb{Z} / 2 \subset \mathfrak{S}_{3}$ the quotient $C_{S} / \mathbb{Z} / 2$ is an elliptic curve. Since all such subgroups are conjugated it is the same elliptic curve. Any elliptic curve (over a field of characteristic $\neq 2$ ) can be obtained in this way: realize it as the double cover of $\mathbb{P}^{1}$ ramified in

$$
\left\{1,(x+1 / x) / 2,(\zeta x+1 / \zeta x) / 2,\left(\zeta^{2} x+1 / \zeta^{2} x\right) / 2\right\}
$$

corresponding to the $\mathfrak{S}_{3}$-orbit

$$
\left\{x, \zeta x, \zeta^{2} x, 1 / x, \zeta / x, \zeta^{2} / x\right\}
$$

where $\zeta$ is a third root of 1 and $x \in \mathbb{P}^{1}$ is an arbitrary point not equal to $0, \infty$ and any cubic root of 1 or -1 . The quotient $C_{S} / \mathbb{Z} / 3$ is rational.

Applying the argument of Corollary 2.4 and endomorphisms (sums of powers of the Frobenius, they commute with the $\mathbb{Z} / 3$-action) we obtain our claim.

Remark 4.6. There exist K3 surfaces that are not generalized Kummer K3 surfaces but are dominated by such. Clearly, they satisfy the conclusion of Corollary 4.5.

Remark 4.7. We do not know whether or not every algebraic K3 surface contains infinitely many rational curves (elliptic K3 surfaces do, see [5]). It is known 
that primitive classes in $\operatorname{Pic}(S)$ of a general K3 surface $S$ over $\mathbb{C}$ are represented by rational curves with at worst nodal singularities (see [26], [7], for example). In particular, a general polarized $S$ with $\operatorname{rk} \operatorname{Pic}(S) \geq 2$ has infinitely many rational curves. See, however, [10] for examples of surfaces with $\operatorname{rk} \operatorname{Pic}\left(S_{\overline{\mathbb{Q}}}\right)=1$.

Remark 4.8. Theorem 4.2 can fail if $k=\overline{\mathbb{F}}_{q}(t)$ as we now show. Let $S_{0}$ be a nonsupersingular Kummer surface over $\overline{\mathbb{F}}_{q}$ (and therefore not uniruled, by Remark 3.4). Let $S$ be a base extension of $S_{0}$ to $k$. Choose a nonrational curve $C_{0}$ in $S_{0}$. View the function field $k_{0}=\overline{\mathbb{F}}_{q}\left(C_{0}\right)$ as a finite extension of $k$. Restricting the diagonal map $C_{0} \rightarrow S_{0} \times C_{0}$ to the generic point gives a point $s \in S\left(k_{0}\right)$. If the conclusion of Theorem 4.2 were valid for $S$, then over some finite extension $k_{0}^{\prime}$ of $k_{0}$, there would be a nonconstant rational curve through $s$ and hence a dominant rational map $\mathbb{P}^{1} \times C_{0}^{\prime} \rightarrow S_{0}$, where $C_{0}^{\prime}$ is a curve over $\overline{\mathbb{F}}_{q}$ with function field $k_{0}^{\prime}$. Therefore, $S_{0}$ is uniruled-contradiction.

5. Surfaces of general type. Using similar ideas we can construct nonuniruled surfaces $S$ of general type over finite fields $k$ with nontrivial Brauer group of finite height [3] such that every algebraic point $s \in S(\bar{k})$ lies on a rational curve and any two points can be connected by a chain of rational curves. (However, the degrees of these curves cannot be bounded, a priori.)

For simplicity, let us assume that $p:=\operatorname{char}(k) \geq 5$. Let $S_{0}$ be a unirational surface of general type over $k$, for example

$$
x^{p+1}+y^{p+1}+z^{p+1}+t^{p+1}=0
$$

([21], Section 5). Let $\mathbb{P}^{2} \rightarrow S_{0}$ be the corresponding (purely inseparable) covering of degree a power of $p$.

Let $S_{1}$ be a nonsupersingular, and therefore, nonuniruled, Kummer K3 surface admitting an abelian cover onto $\mathbb{P}^{2}$ of degree prime to $p$ with Galois group $G$ for example, a double cover (here we may have to enlarge the ground field $k$ ).

Lemma 5.1. For any n coprime to $p$, and any finite purely inseparable extension $L / K$ we have a natural isomorphism, induced by inclusion $K \hookrightarrow L$,

$$
K^{*} /\left(K^{*}\right)^{n}=L^{*} /\left(L^{*}\right)^{n} .
$$

Poof. Indeed, there exists an $m \in \mathbb{N}$ such that $K^{*}$ contains the $p^{m}$-powers of all elements of $L^{*}$. Since $p^{m}$ and $n$ are coprime the claimed isomorphism follows.

Let $L=\bar{k}\left(\mathbb{P}^{2}\right)$. By Kummer theory, the extension of function fields $\bar{k}\left(S_{1}\right)$ over $L$ is obtained by adjoining the $n$th roots of the elements of a finite subset $T$ of $L^{*}$, for some positive integer $n$ prime to $p$. By Lemma 5.1, we may multiply each element of $T$ by an element of $\left(L^{*}\right)^{n}$ in order to assume that $T \subset K^{*}$. Adjoining 
the $n$th roots of the elements of $T$ to $\bar{k}\left(S_{0}\right)$ gives the function field of a surface $S$ over $\bar{k}$. In particular, we have rational maps:

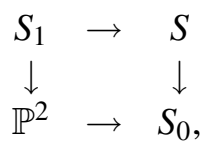

where $S$ is a surface of general type (since the corresponding function field is a separable abelian extension of degree coprime to $p$ ). At the same time there is a surjective purely inseparable map $S_{1} \rightarrow S$. Surjectivity implies that there is a rational curve (defined over $\bar{k}$ ) passing through every algebraic point of $S$, to get every point we may need to pass to a blowup $\tilde{S}_{1}$ of $S_{1}$ resolving the indeterminacy of the dominant map $S_{1} \rightarrow S$ (exceptional curves are rational over $\bar{k}$ ). By pure inseparability, if we had a dominant map $C \times \mathbb{P}^{1} \rightarrow S$ then we would also have a dominant map $C \times \mathbb{P}^{1} \rightarrow S_{1}$ (seen on the level of function fields), contradicting the assumption that $S_{1}$ is not uniruled.

6. Higher dimensions. Arguments as in the proof of Theorem 4.2 give us the following result: Let $k$ be a finite field, $C$ a hyperelliptic curve of genus $\geq 2$ over $k, J$ its Jacobian, $\sigma$ the standard involution on $J$ and $S=J / \sigma$ the associated Kummer variety. Then every rational point $s \in S(\bar{k})$ lies on some rational curve defined over $\bar{k}$. Similar results hold for some other classes of nonuniruled higherdimensional varieties.

Definition 6.1. A smooth projective variety $V$ is called Calabi-Yau if its canonical class is trivial and $h^{0}\left(\Omega_{V}^{i}\right)=0$ for all $i=1, \ldots, \operatorname{dim} X-1$.

Example 6.2. Let $E$ an elliptic curve over $k$ with an automorphism $\rho$ of order 3 and $A:=E^{3}$. The quotient $A / \rho$ (diagonal action) admits an desingularization $V$ which is a Calabi-Yau variety.

There are many embeddings $\iota: E \hookrightarrow A$ and, in particular, every torsion point in $A$ lies on some $\iota(E)$.

If $k$ is finite then every point in $V(\bar{k})$ lies on some $\bar{k}$-rational curve in $V$. Moreover, $E^{2} / \rho$ (diagonal action) is a rational surface. Hence every point in $V(\bar{k})$ lies in fact on a rational surface defined over $\bar{k}$.

Example 6.3. Let $C$ be the Klein quartic curve and $J$ its Jacobian. Then the quotient of $J / \sigma$, where $\sigma$ is an automorphism of order 7, admits a desingularization $V$ which is a Calabi-Yau threefold (see [15], for example). Again, over finite fields, one can show that every algebraic point of $V$ lies on a rational curve.

Example 6.4. The following varieties have been considered in [24]: Let $S$ be a K3 surface with an involution $\sigma$ and $E$ an elliptic curve with the standard involution $\tau$. There exists a nonsingular model $V$ of $E \times S /(\tau \times \sigma)$, which is a 
Calabi-Yau threefold. If we choose $S$ and $E$, defined over a finite field, so that every algebraic point of $S$ lies on a rational curve, then the same property holds for $V$.

Conjecture 6.5. Let $X$ be any smooth projective variety over a finite field $k$. Assume that $X$ has trivial canonical class and that $X_{\bar{k}}$ has trivial algebraic fundamental group. Then every algebraic point of $X$ lies on a rational curve $C \subset X$, defined over $\bar{k}$.

Remark 6.6. If $A$ is a general abelian variety of dimension $n \geq 3$ (over $\mathbb{C}$ or over an algebraic closure of $\left.\overline{\mathbb{F}}_{q}(x)\right)$ and $\sigma$ is the standard involution, then $A / \sigma$ contains no rational curves, has trivial fundamental group and has Kodaira dimension zero (see Remark 2.1). However, the canonical class of a desingularization is nontrivial, for $n \geq 3$. This also shows that the presence of rational curves is highly unstable under deformations.

An interesting test of Conjecture 6.5 would be the case of a smooth quintic in $\mathbb{P}^{4}$.

DePartment of Mathematics, Courant Institute of Mathematical Sciences, New YORK UNIVERSITY, NEW YORK, NY

Mathematisches InSTitut, BunSEnSTR. 3-5, D-37073 GötTINGEN, GeRMANY

REFERENCES

[1] G. W. Anderson and R. Indik, On primes of degree one in function fields, Proc. Amer. Math. Soc. 94 (1985), 31-32.

[2] C. Araujo and J. Kollár, Rational curves on varieties, Higher Dimensional Varieties and Rational Points (Budapest, 2001), Bolyai Soc. Math. Stud., vol. 12, Springer-Verlag, Berlin, 2003, pp. 13-68.

[3] M. Artin, Supersingular K3 surfaces, Ann. Sci. École Norm. Sup. (4) 7 (1974), 543-567.

[4] A. Beauville, Counting rational curves on K3 surfaces, Duke Math. J. 97 (1999), 99-108.

[5] F. Bogomolov and Y. Tschinkel, Density of rational points on elliptic K3 surfaces, Asian J. Math. 4 (2000), 351-368.

[6] J. Bryan and N. C. Leung, The enumerative geometry of $K 3$ surfaces and modular forms, J. Amer. Math. Soc. 13 (2000), 371-410.

[7] X. Chen, A simple proof that rational curves on $K 3$ are nodal, Math. Ann. 324 (2002), 71-104.

[8] F. R. Cossec and I. V. Dolgachev, Enriques Surfaces. I, Progr. Math., vol. 76, Birkhäuser, Boston, MA, 1989.

[9] S. J. Edixhoven, B. J. J. Moonen and F. Oort, Open problems in algebraic geometry, Bull. Sci. Math. 125 (2001), 1-22.

[10] J. S. Ellenberg, $K 3$ surfaces over number fields with geometric Picard number one, Arithmetic of Higherdimensional Algebraic Varieties (Palo Alto, CA, 2002), Progr. Math., vol. 226, Birkhäuser, Boston, MA, 2004, pp. 135-140

[11] T. Katsura, Generalized Kummer surfaces and their unirationality in characteristic p, J. Fac. Sci. Univ. Tokyo Sect. IA Math. 34 (1987), 1-41. 
[12] N. M. Katz, Space filling curves over finite fields, Math. Res. Lett. 6 (1999), 613-624.

[13] Twisted L-functions and Monodromy, Ann. Math. Stud., vol. 150, Princeton University Press, Princeton, NJ, 2002.

[14] D. Mumford, Abelian Varieties, Tata Inst. Fund. Res. Stud. Math., no. 5, Published for the Tata Institute of Fundamental Research, Bombay, 1970.

[15] K. Oguiso, On the complete classification of Calabi-Yau threefolds of type $\mathrm{III}_{0}$, Higher-dimensional Complex Varieties (Trento, 1994), de Gruyter, Berlin, 1996, pp. 329-339.

[16] F. Oort and J. de Jong, Hyperelliptic curves in abelian varieties, J. Math. Sci. 82 (1996), 3211-3219.

[17] G. P. Pirola, Curves on generic Kummer varieties, Duke Math. J. 59 (1989), 701-708.

[18] B. Poonen, Néron-Tate projection of algebraic points, Internat. Math. Res. Notices (2001), 435-440.

[19] F. Pop and M. Saidi, On the specialization homomorphism of fundamental groups of curves in positive characteristic, Galois groups and Fundamental Groups (L. Schneps, eds.), Math Sci. Res. Inst. Publ., vol. 41, Cambridge Univ. Press, 2003, pp. 107-118.

[20] A. N. Rudakov and I. R. Shafarevich, Supersingular $K 3$ surfaces over fields of characteristic 2, Izv. Akad. Nauk SSSR Ser. Mat. 42 (1978), 848-869.

[21] Surfaces of type $K 3$ over fields of finite characteristic, Current Problems in Math., vol. 18, Akad. Nauk SSSR, 1981, pp. 115-207.

[22] J.-P. Serre, Lectures on the Mordell-Weil Theorem, 3rd ed., Aspects Math., Friedr. Vieweg \& Sohn, Braunschweig, 1997.

[23] T. Shioda, Some results on unirationality of algebraic surfaces, Math. Ann. 230 (1977), 153-168.

[24] C. Voisin, Miroirs et involutions sur les surfaces K3, Astérisque (1993), 273-323.

[25] A. Weil, Zum Beweis des Torellischen Satzes, Nachr. Akad. Wiss. Göttingen. Math.-Phys. Kl. IIa. 1957 (1957), 33-53.

[26] S.-T. Yau and E. Zaslow, BPS states, string duality, and nodal curves on K3, Nuclear Phys. B 471 (1996), 503-512. 